\title{
Associations of the Glycaemic Control of Diabetes with Dementia and Physical Function in Rural-Dwelling Older Chinese Adults: A Population-Based Study
}

\author{
Yanhong Jia (iD) ${ }^{1-3, *}$ \\ Rui Liu' ${ }^{1} *$ \\ Shi Tang 1,2,4 \\ Dongming Zhang ${ }^{5}$ \\ Yongxiang Wang ${ }^{1,2,4}$ \\ Lin Cong ${ }^{1,2,4}$ \\ Tingting Hou ${ }^{1,2,4}$ \\ Juan Ren' \\ Yifeng Du (iD) ${ }^{1,2,4}$
}

'Department of Neurology, Shandong Provincial Hospital, Cheeloo College of Medicine, Shandong University, Jinan, Shandong, People's Republic of China; 2Department of Neurology, Shandong Provincial Hospital Affiliated to Shandong First Medical University, Jinan, Shandong, People's Republic of China; ${ }^{3}$ Department of Neurology, Baotou Central Hospital, Baotou, Inner Mongolia, People's Republic of China; ${ }^{4}$ Shandong Provincial Clinical Research Center for Neurological Diseases, Jinan, Shandong, People's Republic of China; ${ }^{5}$ Department of General Surgery, Baotou Central Hospital, Baotou, Inner Mongolia, People's Republic of China

*These authors contributed equally to this work

\begin{abstract}
Purpose: To examine the associations of impaired fasting glucose (IFG) and glycaemic control of diabetes with dementia, global cognitive function and physical function among rural-dwelling Chinese older adults.
\end{abstract}

Patients and Methods: This population-based cross-sectional study included 4583 participants (age $\geq 65$ years, $57.3 \%$ women) living in Yanlou Town, Yanggu County, western Shandong Province, China. In 2018, data were collected through interviews, clinical examinations, neuropsychological tests, and laboratory tests. Diabetes status was defined by selfreported physician-diagnosed diabetes, current use of antidiabetic agents, and fasting blood glucose tests. Global cognitive function was assessed using the Mini-Mental State Examination. Dementia was diagnosed following DSM-IV criteria, and Alzheimer's disease (AD) was diagnosed following the National Institute on Aging-Alzheimer's Association criteria. Physical function was assessed by the Short Physical Performance Battery. Data were analysed using multiple logistic and general linear regression models.

Results: IFG was found in 267 participants, and diabetes was diagnosed in 658 participants (257 with well-controlled diabetes, 401 with poorly controlled diabetes). Dementia was diagnosed in 166 participants (116 with $\mathrm{AD}$ ), and physical functional impairment was found in 1973 participants. The multi-adjusted odds ratio (OR) of dementia associated with poorly controlled diabetes (vs without IFG or diabetes) was 2.41 (95\% CI 1.52-3.84), and the $\mathrm{OR}$ of $\mathrm{AD}$ associated with poorly controlled diabetes was 2.32 (1.34-4.04). In addition, the adjusted OR of physical functional impairment was $1.40(1.06-1.85)$ for wellcontrolled diabetes and $1.69(1.35-2.12)$ for poorly controlled diabetes. However, IFG was not associated with cognitive or physical function.

Conclusion: The glycaemic control status of diabetes patients was associated with cognitive impairment and physical functional impairment.

Keywords: impaired fasting glucose, Alzheimer's disease, cognitive function, physical functional impairment

\section{Introduction}

The global prevalence of diabetes in adults is $8.4 \%$; it affected 451 million adults in 2017, and that number is expected to increase to nearly 693 million by 2045. People with diabetes were significantly more often burdened with multimorbidity and polypharmacy, placing a huge economic burden on society. ${ }^{1,2}$ China has the world's 
largest diabetes epidemic, and the prevalence of diabetes among people 60 years or older is $21.7 \%$. Approximately $70.4 \%$ of rural-dwelling people with diabetes are not treated, and $56.6 \%$ of treated people do not have adequate glycaemic control, which was significantly higher than that among urban-dwelling people with diabetes. ${ }^{3}$ In addition, as a high-risk state for developing diabetes, impaired fasting glucose (IFG) has received increasing attention. ${ }^{4}$ A meta-analysis showed that annualized rates of progression to diabetes in patients with isolated IFG were $6 \%-9 \%$, and compared to normoglycemic people, the overall risk of developing diabetes in studies of people with IFG was $7.54 .^{5}$ The increased age profile of people with IFG and diabetes implies that they may have a higher prevalence of cognitive impairment and physical disability and a lower quality of life.

There has been a growing body of population-based studies on diabetes and cognitive health. Many studies have suggested that diabetes is associated with all-cause dementia and Alzheimer's disease (AD) ${ }^{6-8}$ There is evidence of a shared pathogenesis between diabetes and $\mathrm{AD}$, which has been termed type 3 diabetes. ${ }^{9,10}$ However, population-based studies on the associations of IFG and glycaemic control of diabetes with dementia are sparse. Furthermore, the previously published studies were limited by small sample sizes, case-control designs, selection bias, or neuropsychological test performance but not accurate dementia diagnosis. ${ }^{11,12}$

In addition to cognitive function, physical function is also an important aspect that affects the quality of life of older adults, acting as a strong predictor of mortality, hospitalization, and disability. ${ }^{13}$ Several studies have shown that diabetes is associated with worse physical functioning among older adults. ${ }^{14,15}$ The Elderly Health Centres (EHC) study of 66,813 community-dwelling participants in Hong Kong aged 65 years or older found that those receiving regular treatment for diabetes were 1.7 times more likely to have physical functional impairment than those without diabetes. ${ }^{16}$ However, this study only adjusted for age, sex, and educational level as covariates and did not take other potential confounders, such as lifestyle factors or comorbidities, into account. Moreover, few studies have analysed the associations of IFG or glycaemic control of diabetes with physical functioning.

Therefore, in this population-based cross-sectional study of older adults living in rural communities in China, we sought to evaluate whether IFG and glycaemic control of diabetes are associated with dementia, AD, global cognitive function, and physical functional impairment. We hypothesized that worse glycaemic control of diabetes is associated with a higher likelihood of dementia and physical functional impairment in older adults.

\section{Materials and Methods Study Design and Participants}

Participants in this cross-sectional population-based study were derived from the baseline assessments of the Multimodal Intervention to Delay Dementia and Disability in Rural China (MIND-China), ${ }^{17,18}$ a participating project within the World-Wide FINGERS Network. ${ }^{19}$ MINDChina targeted residents aged $\geq 60$ years who were living in the rural communities of Yanlou Town, Yanggu County in western Shandong Province, China. From March to September 2018, 5765 eligible participants were recruited. Because we focused on late-onset dementia and physical functional impairment, we excluded people who were aged 60-64 years $(\mathrm{n}=519)$ from this study. Among these participants, 46 were further excluded due to major depression disorders or other severe psychiatric diseases, 224 were excluded due to missing data on the Mini-Mental State Examination (MMSE) score, and 393 were further excluded due to missing data on the Short Physical Performance Battery (SPPB), leaving 4583 participants for the current analysis. Compared to participants who were aged $\geq 65$ years and excluded from our analyses, participants included in the analytical sample were younger (mean age, $71.32 \mathrm{vs}$ 74.66 years, $p<0.001$ ), but the two groups did not differ significantly by sex (women: $57.3 \%$ vs $56.0 \%, p=0.501$ ).

MIND-China study was conducted in accordance with the Declaration of Helsinki, and has been approved by the Ethics Committee of Shandong Provincial Hospital in Jinan, Shandong. Written informed consent was obtained from all participants, or in the case of severely cognitively impaired participants, from informants. MIND-China was registered in the Chinese Clinical Trial Registry (registration no.: ChiCTR1800017758).

\section{Data Collection}

Data were collected through face-to-face interviews, clinical examinations, neuropsychological tests, and laboratory tests by trained medical staff. ${ }^{17,18}$ Following a structured questionnaire, we collected data on sociodemographics (for example, age, sex, and education), lifestyle factors (for example, alcohol consumption and smoking), medical history (for example, hypertension, diabetes, coronary heart 
disease, and stroke), use of medications, cognitive function, and physical function. All medications were classified according to the Anatomical Therapeutic Chemical (ATC) system. Weight and height were measured in light clothing without shoes. Body mass index (BMI) was calculated as weight (in kilograms) divided by height (in metres) squared. Arterial blood pressure was measured on the right upper arm after a 5-minute rest in a sitting position using an electronic sphygmomanometer (Omron HEM-7127J; Omron Corporation, Kyoto, Japan). The 12-lead resting electrocardiogram was recorded using an electrocardiograph (CM300, COMEN, Shenzhen, China). Peripheral blood samples were collected after an overnight fast by experienced nurses. Fasting blood glucose, total cholesterol (TC), triglycerides (TG), low-density lipoprotein cholesterol (LDL-C), and high-density lipoprotein cholesterol (HDL-C) were measured using an Automatic Biochemical Analyser (CS-600B, DIRUI Corporation, Changchun, China) at the Yanlou Town Hospital laboratory using enzymatic methods. Genomic DNA was extracted from peripheral blood leukocytes, and the apolipoprotein $\mathrm{E}(A P O E)$ genotype was detected using multiple-polymerase chain reaction amplification (iGeneTech Bioscience Co., Ltd, Beijing, China).

\section{Definitions of IFG and Diabetes}

Diabetes was defined as a fasting blood glucose $\geq 7.0$ $\mathrm{mmol} / \mathrm{L}$, having a self-reported previous diagnosis of diabetes by a physician, or current use of antidiabetic agents (ATC code A10). Participants who did not have diabetes but had a fasting blood glucose level of 6.1-6.9 mmol/L were considered to have IFG. Both fasting blood glucose cut-off points followed the World Health Organization and the International Diabetes Federation Standards. ${ }^{20}$

Well-controlled diabetes was defined as diabetes patients with a fasting blood glucose $<7.0 \mathrm{mmol} / \mathrm{L}$, and poorly controlled diabetes was defined as diabetes patients with a fasting blood glucose $\geq 7.0 \mathrm{mmol} / \mathrm{L}$.

\section{Assessment of Cognitive Function, Diagnosis of Dementia and Dementia Subtypes}

A neuropsychological test battery, mainly including the MMSE, Ascertain Dementia 8-item Questionnaire, Auditory Verbal Learning Test, Category Verbal Fluency Test, Forward and Backward Digit Span Test, and Trail Making Test A and B, was used to assess cognitive function, including subjective cognitive complaints, global cognitive function, and cognitive domains (memory, language, attention, and executive function). We used the Chinese version of the MMSE to evaluate global cognitive function. ${ }^{21}$ A lower MMSE score indicates worse global cognitive performance.

A three-step diagnostic procedure was followed to make a diagnosis of dementia and subtypes of dementia. First, trained clinicians and interviewers conducted routine clinical examinations and comprehensive assessments for each participant following structured questionnaires. The assessments included medical history, a neuropsychological test battery, and activities of daily living (ADLs). Second, neurologists specializing in dementia diagnosis and care reviewed all of the data and made a preliminary diagnosis for participants who were suspected of having dementia. Finally, the neurologists conducted the second face-to-face interviews with those participants who were suspicious of having dementia, and reassessed their medical history, cognitive status, ADLs, and whenever available, neuroimaging data. If the participants were not able to participate in the interview due to severe cognitive impairment or were not available for the face-to-face interviews (approximately $13 \%$ ), the neurologists interviewed their family members, neighbours, or their village doctors (who provide primary care services to the local residents). Based on all of the data, a diagnosis of dementia was made according to the Diagnostic and Statistical Manual of Mental Disorders, 4th Edition (DSM-IV) criteria. ${ }^{22}$ In cases of uncertainty, a senior neurologist was consulted, and a consensus diagnosis was made. Dementia was further classified into AD following the National Institute on Ageing-Alzheimer's Association (NIAAA) criteria, ${ }^{23}$ and vascular dementia (VaD) following the National Institute of Neurological Disorders and Stroke and the Association Internationale pour la Recherche et l'Enseignement en Neurosciences (NINDS-AIREN) criteria. ${ }^{24}$ Demented participants who could not be diagnosed with $\mathrm{AD}$ or $\mathrm{VaD}$ were classified as having other types of dementia.

\section{Assessment of Physical Function}

Physical function was assessed by the SPPB. ${ }^{25}$ It is a widely used measure of lower extremity physical function. It consists of three measurements: standing balance, repeated chair stands, and a 4-metre walk, and each domain is scored from $0-4$. The summary score of the balance score, chair score, and walk score was used for the analyses. A lower SPPB score indicates worse physical function. Physical functional impairment was considered 
a score of 9 or less out of a total score of 12 according to previous studies. ${ }^{26}$

\section{Assessment of Covariates}

BMI $\left(\mathrm{kg} / \mathrm{m}^{2}\right)$ was categorized as underweight $(<18.5)$, normal (18.5-23.9), overweight (24-27.9), and obese ( $\geq 28$ ), following the criteria for the Chinese population. ${ }^{27}$ Smoking status and alcohol consumption were categorized as never, previous, and current, respectively. Hypertension was defined as blood pressure $\geq 140 / 90 \mathrm{mmHg}$ or current use of antihypertensive drugs (ATC codes $\mathrm{C} 02, \mathrm{C} 03$, and C07-C09). Dyslipidemia was defined as TC $\geq 6.22 \mathrm{mmol} /$ $\mathrm{L}$, or $\mathrm{TG} \geq 2.27 \mathrm{mmol} / \mathrm{L}$, or HDL-C $<1.04 \mathrm{mmol} / \mathrm{L}$, or LDL-C $\geq 4.14 \mathrm{mmol} / \mathrm{L}$, or the use of lipid-regulating drugs (ATC code C10). Coronary heart disease was defined according to self-reported history or electrocardiogram examination, including angina, myocardial infarction, history of coronary angioplasty, and history of coronary artery bypass grafting. $A P O E$ genotype was dichotomized as carriers and non-carriers of the $A P O E \& 4$ allele.

\section{Statistical Analysis}

We reported frequencies (percentages) for categorical variables and means (standard deviations, SDs) for continuous variables. Characteristics of the study participants by IFG and diabetes status were compared using the KruskalWallis $H$-test for continuous variables and the chi-square test for categorical variables. Pairwise comparisons among the IFG and glycaemic control status of diabetes were made using Bonferroni adjustment in post hoc analyses. Binary logistic regression models were used to examine the associations of IFG and glycaemic control status of diabetes with dementia and $\mathrm{AD}$. $\mathrm{VaD}(\mathrm{n}=42)$ and other types of dementia $(n=8)$ were not analysed separately because there were relatively few cases. General linear regression models were used to analyse the associations of IFG and glycaemic control status of diabetes with MMSE score, total SPPB score, balance score, chair score, and walk score.

We reported the results from two models: Model 1 was adjusted for age and sex, and Model 2 was additionally adjusted for education, BMI, alcohol consumption, smoking status, hypertension, dyslipidemia, coronary heart disease, and stroke. When analysing the associations of IFG and glycaemic control status of diabetes with dementia, $\mathrm{AD}$, and global cognitive performance, we additionally adjusted for the APOE genotype in Model 2.
IBM SPSS Statistics 22.0 (IBM SPSS Inc., Chicago, Illinois) was used for all analyses. Statistical significance was set at two-tailed $p<0.05$.

\section{Results}

\section{Characteristics of the Study Participants}

The mean age of the 4583 participants was 71.32 (SD, 5.00; range, 65-93) years, $57.3 \%$ were women, and their average years of formal schooling was 3.21 years (SD, 3.44; range, 0-19, 38.8\% illiteracy). Among those, 267 $(5.8 \%)$ were diagnosed with IFG, and $658(14.4 \%)$ were diagnosed with diabetes, including 257 (5.6\%) with wellcontrolled diabetes and $401(8.7 \%)$ with poorly controlled diabetes. For cognitive function and physical function, 166 (3.6\%) were diagnosed with dementia, including 116 (2.5\%) with $\mathrm{AD}, 42(0.9 \%)$ with $\mathrm{VaD}$, and $8(0.2 \%)$ with other types of dementia. 1973 (43.1\%) were found to have physical functional impairment.

Compared with participants without IFG or diabetes, participants with IFG were more likely to be obese, had a higher prevalence of hypertension, dyslipidemia and coronary heart disease $(p<0.05)$. Compared with participants without IFG or diabetes, participants with wellcontrolled diabetes were more likely to be women and obese, less likely to drink alcohol or smoke, and had a higher prevalence of hypertension, coronary heart disease, stroke, and physical functional impairment. In addition, they had lower total SPPB scores, balance scores, chair scores, and walk scores $(p<0.05)$. Compared with participants without IFG or diabetes, participants with poorly controlled diabetes were younger; more likely to be obese; less likely to drink alcohol; had a higher prevalence of dyslipidemia, coronary heart disease, stroke, dementia, and physical functional impairment; and had a lower total SPPB score, balance score, chair score, and walk score $(p<0.05)$. The four groups had no significant differences in the average years of formal schooling, APOE $\varepsilon 4$ status, or MMSE score $(p>0.05)$ (Table 1).

\section{Associations of IFG and Diabetes with Dementia and Global Cognitive Function}

After adjusting for age and sex, poorly controlled diabetes was significantly associated with a higher likelihood of all-cause dementia (Table 2, Model 1). The associations remained statistically significant when additionally adjusted for multiple confounders, such 
Table I Characteristics of the Study Participants

\begin{tabular}{|c|c|c|c|c|c|}
\hline Characteristics & $\begin{array}{l}\text { Without IFG or } \\
\text { Diabetes }(n=3658)\end{array}$ & IFG (n = 267) & $\begin{array}{l}\text { Well-Controlled } \\
\text { Diabetes }(n=257)\end{array}$ & $\begin{array}{l}\text { Poorly Controlled } \\
\text { Diabetes }(n=401)\end{array}$ & $p$ \\
\hline Age (years) & $71.50(5.08)^{c}$ & $71.15(5.02)$ & $70.48(4.23)$ & $70.30(4.58)^{c}$ & $<0.001$ \\
\hline Women, n (\%) & $2040(55.8)^{\mathrm{b}}$ & $156(58.4)^{d}$ & $186(72.4)^{b, d, f}$ & $246(61.3)^{f}$ & $<0.001$ \\
\hline Education (years) & $3.23(3.44)$ & $3.04(3.35)$ & $3.05(3.38)$ & $3.25(3.50)$ & 0.755 \\
\hline 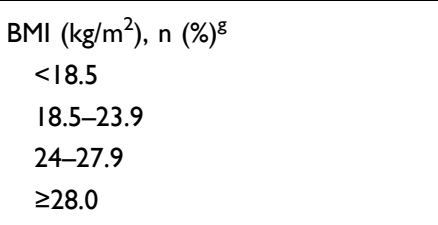 & $\begin{array}{l}\mid 30(3.6) \\
\mid 498(4 \mid .2)^{\mathrm{a}, \mathrm{b}, \mathrm{c}} \\
|37|(37.7) \\
64 \mid(\mid 7.6)^{\mathrm{a}, \mathrm{b}, \mathrm{c}}\end{array}$ & $\begin{array}{l}5(1.9) \\
69(25.8)^{\mathrm{a}} \\
113(42.3) \\
80(30.0)^{\mathrm{a}}\end{array}$ & $\begin{array}{l}4(1.6) \\
64(25.1)^{\mathrm{b}} \\
108(42.4) \\
79(31.0)^{\mathrm{b}}\end{array}$ & $\begin{array}{l}7(1.8) \\
119(29.9)^{c} \\
168(42.2) \\
104(26.1)^{c}\end{array}$ & $<0.001$ \\
\hline $\begin{array}{l}\text { Alcohol consumption, n (\%) } \\
\text { Never } \\
\text { Previous } \\
\text { Current }\end{array}$ & $\begin{array}{l}2184(59.7)^{\mathrm{b}} \\
362(9.9) \\
1 \mathrm{II} 2(30.4)^{\mathrm{b}, \mathrm{c}}\end{array}$ & $\begin{array}{l}156(58.4)^{d} \\
22(8.2) \\
89(33.3)^{d, e}\end{array}$ & $\begin{array}{l}187(72.8)^{\mathrm{b}, \mathrm{d}} \\
19(7.4) \\
5 \mathrm{I}(19.8)^{\mathrm{b}, \mathrm{d}}\end{array}$ & $\begin{array}{l}265(66.1) \\
45(11.2) \\
91(22.7)^{c, e}\end{array}$ & $<0.001$ \\
\hline $\begin{array}{l}\text { Smoking, n (\%) } \\
\text { Never } \\
\text { Previous } \\
\text { Current }\end{array}$ & $\begin{array}{l}2292(62.7)^{\mathrm{b}} \\
804(22.0)^{\mathrm{b}, \mathrm{c}} \\
562(15.4)\end{array}$ & $\begin{array}{l}167(62.5)^{d} \\
63(23.6)^{d, e} \\
37(13.9)\end{array}$ & $\begin{array}{l}204(79.4)^{\mathrm{b}, \mathrm{d}, \mathrm{f}} \\
27(10.5)^{\mathrm{b}, \mathrm{d}} \\
26(10.1)^{\mathrm{f}}\end{array}$ & $\begin{array}{l}275(68.6)^{f} \\
52(13.0)^{c, e} \\
74(18.5)^{f}\end{array}$ & $<0.001$ \\
\hline Fasting blood glucose $(\mathrm{mmol} / \mathrm{L})$ & $5.10(0.44)^{\mathrm{a}, \mathrm{b}, \mathrm{c}}$ & $6.44(0.25)^{\mathrm{a}, \mathrm{d}, \mathrm{e}}$ & $5.85(0.7 \mathrm{I})^{\mathrm{b}, \mathrm{d}, \mathrm{f}}$ & $9.07(2.4 I)^{\mathrm{c}, \mathrm{e}, \mathrm{f}}$ & $<0.001$ \\
\hline Hypertension, $n(\%)^{g}$ & $2359(65 . I)^{a, b}$ & $195(73.3)^{\mathrm{a}}$ & $190(73.9)^{\mathrm{b}}$ & $275(69.3)$ & 0.001 \\
\hline Dyslipidemia, n (\%) & $761(20.8)^{\mathrm{a}, \mathrm{c}}$ & $96(36.0)^{\mathrm{a}, \mathrm{d}}$ & $68(26.5)^{\mathrm{d}, \mathrm{f}}$ & $150(37.4)^{\mathrm{c}, \mathrm{f}}$ & $<0.001$ \\
\hline Coronary heart disease, $\mathrm{n}(\%)$ & $722(19.7)^{\mathrm{a}, \mathrm{b}, \mathrm{c}}$ & $73(27.3)^{\mathrm{a}}$ & $88(34.2)^{b}$ & $111(27.7)^{c}$ & $<0.001$ \\
\hline Stroke, n (\%) & $508(13.9)^{\mathrm{b}, \mathrm{c}}$ & $48(18.0)$ & $69(26.8)^{\mathrm{b}}$ & $83(20.7)^{c}$ & $<0.001$ \\
\hline APOE $\varepsilon 4$ carrier, $\mathrm{n}(\%)^{\mathrm{g}}$ & $584(16.5)$ & $37(14.5)$ & $35(14.1)$ & $54(14.0)$ & 0.420 \\
\hline Dementia, n (\%) & $119(3.3)^{c}$ & $11(4.1)$ & $8(3.1)$ & $28(7.0)^{\mathrm{c}}$ & 0.002 \\
\hline MMSE score & $20.86(6.06)$ & $20.49(6.30)$ & $20.25(6.20)$ & $20.38(6.40)$ & 0.277 \\
\hline $\begin{array}{l}\text { SPPB score } \\
\text { Balance score } \\
\text { Chair score } \\
\text { Walk score }\end{array}$ & $\begin{array}{l}9.32(2.76)^{b, c} \\
3.40(1.07)^{b, c} \\
2.57(1.35)^{b, c} \\
3.35(0.92)^{b, c}\end{array}$ & $\begin{array}{l}9.06(3.02) \\
3.25(1.23) \\
2.51(1.36) \\
3.29(1.00)\end{array}$ & $\begin{array}{l}8.67(2.87)^{b} \\
3.25(1.14)^{b} \\
2.25(1.38)^{b} \\
3.18(1.01)^{b}\end{array}$ & $\begin{array}{l}8.55(3.28)^{c} \\
3.15(1.30)^{c} \\
2.30(1.44)^{c} \\
3.10(1.13)^{c}\end{array}$ & $\begin{array}{l}<0.001 \\
<0.001 \\
<0.001 \\
<0.001\end{array}$ \\
\hline Physical functional impairment, n (\%) & $1510(41.3)^{b, c}$ & $1 / 4(42.7)^{\mathrm{d}, \mathrm{e}}$ & $136(52.9)^{\mathrm{b}, \mathrm{d}}$ & $213(53.1)^{\mathrm{c}, \mathrm{e}}$ & $<0.001$ \\
\hline
\end{tabular}

Notes: Data are mean (standard deviation), unless otherwise specified. Being statistically significant in pairwise comparisons: ${ }^{a}$ Without diabetes or IFG vs IFG. ${ }^{\text {b Without }}$ diabetes or IFG vs Well-controlled diabetes. 'Without diabetes or IFG vs Poorly controlled diabetes. dFG vs Well-controlled diabetes. IFG vs Poorly controlled diabetes. ${ }^{f}$ Well-controlled diabetes vs Poorly controlled diabetes. ${ }^{g}$ Numbers of subjects with missing values are 23 for BMI, 37 for hypertension, and I43 for APOE genotype. In subsequent analyses, missing data of each covariates were created as a dummy variable.

Abbreviations: APOE, apolipoprotein E; BMI, body mass index; IFG, impaired fasting glucose; MMSE, Mini-Mental State Examination; SPPB, Short Physical Performance Battery.

as education, BMI, lifestyle factors, health conditions, and $A P O E$ genotype (Table 2, Model 2). There were no significant associations of IFG or well-controlled diabetes with dementia. The associations of IFG and glycaemic control status of diabetes with AD were consistent with the associations of IFG and glycaemic control status of diabetes with all-cause dementia.
There were no significant associations of IFG or diabetes with MMSE score after adjusting for age and sex (Table 3, Model 1). When additionally adjusted for multiple confounders, poorly controlled diabetes was significantly correlated with a lower MMSE score (Table 3, Model 2). IFG and well-controlled diabetes had no association with the MMSE score. 


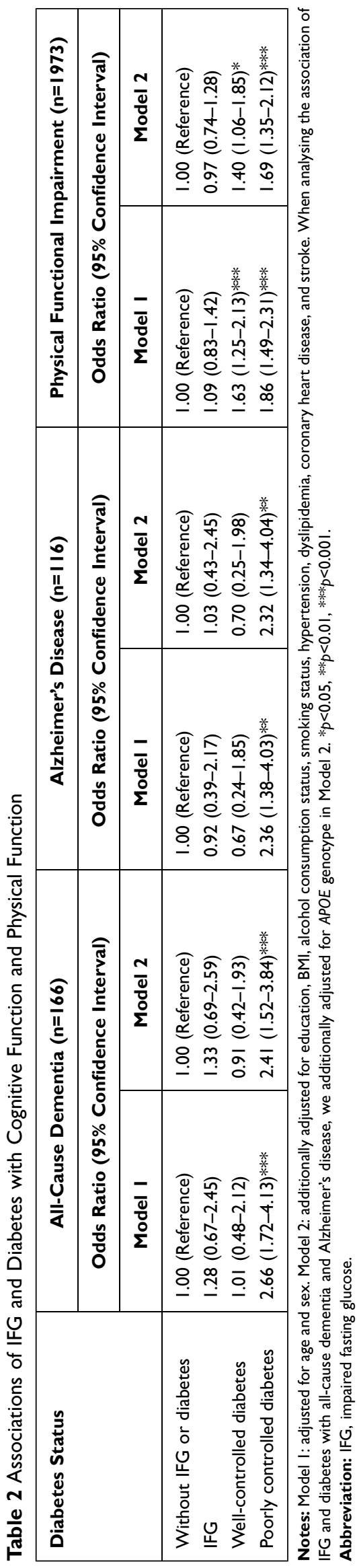

Table 3 Associations of IFG and Diabetes with MMSE Score

\begin{tabular}{|l|l|l|}
\hline \multirow{2}{*}{ Diabetes Status } & \multicolumn{2}{|c|}{$\boldsymbol{\beta}$ Coefficient (95\% Confidence Interval) } \\
\cline { 2 - 3 } & \multicolumn{1}{|c|}{ Model I } & \multicolumn{1}{c|}{ Model 2 } \\
\hline Without IFG or diabetes & 0.00 (Reference) & 0.00 (Reference) \\
IFG & $-0.30(-0.96-0.35)$ & $-0.38(-0.94-0.18)$ \\
Well-controlled diabetes & $0.07(-0.60-0.75)$ & $-0.39(-0.97-0.19)$ \\
Poorly controlled diabetes & $-0.47(-1.01-0.08)$ & $-0.67(-1.14--0.20) * *$ \\
\hline
\end{tabular}

Notes: Model I: adjusted for age and sex. Model 2: additionally adjusted for education, BMI, alcohol consumption status, smoking status, hypertension, dyslipidemia, coronary heart disease, stroke, and APOE genotype. $* *$ $p<0.01$.

Abbreviations: IFG, impaired fasting glucose; MMSE, Mini-Mental State Examination.

\section{Associations of IFG and Diabetes with Physical Functioning}

After controlling for age and sex, well-controlled and poorly controlled diabetes were both significantly associated with physical functional impairment (Table 2, Model 1). In the multi-adjusted models, the associations remained statistically significant (Table 2, Model 2). There was no association between IFG and physical functional impairment.

Well-controlled and poorly controlled diabetes were both associated with a lower balance score, chair score, walk score, and total SPPB score when adjusted for age and sex (Table 4, Model 1). When additionally adjusted for multiple confounders, the associations of wellcontrolled and poorly controlled diabetes with a lower chair score and total SPPB score remained statistically significant. However, in the multi-adjusted models, poorly controlled but not well-controlled diabetes was associated with lower balance score and walk score (Table 4, Model 2). After controlling for age and sex, IFG was associated with a lower balance score (Table 4, Model 1). However, when additionally adjusted for multiple confounders, the association became nonsignificant (Table 4, Model 2). There was no association of IFG with chair score, walk score, or total SPPB score.

\section{Discussion}

In this population-based cross-sectional study that targeted rural community-dwelling older adults in China, we found that poorly controlled diabetes was associated with a higher likelihood of all-cause dementia and $\mathrm{AD}$ and worse global cognitive performance. Both well-controlled and poorly controlled diabetes were associated with a higher likelihood of physical functional impairment. This study draws attention to the fact that rural 


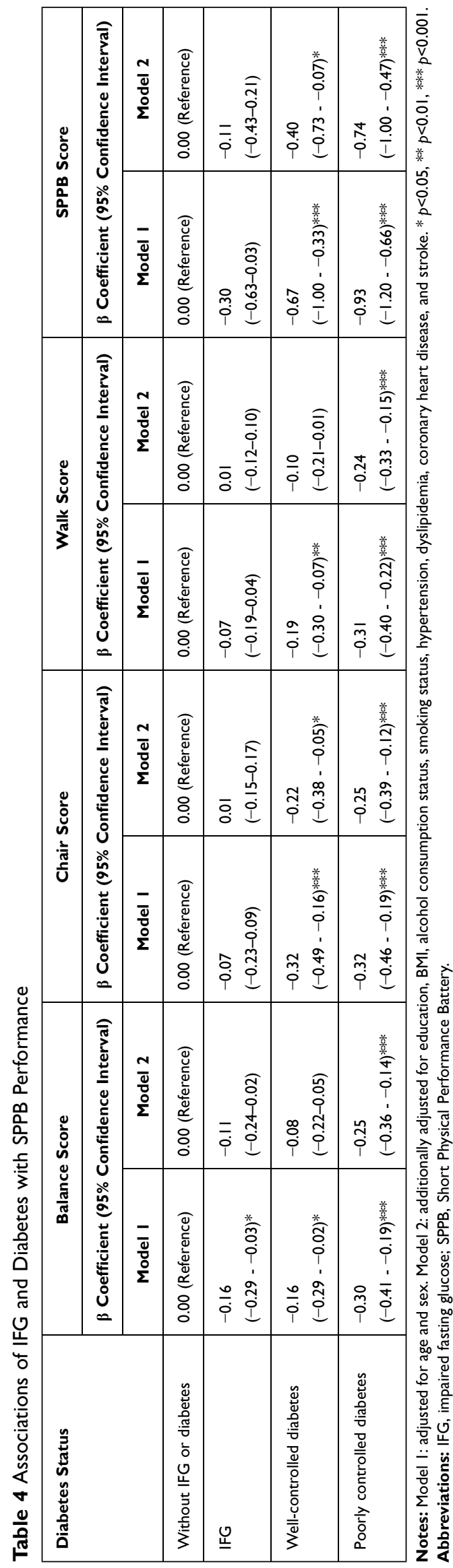

community-dwelling older people with diabetes, especially poorly controlled diabetes, have a higher likelihood of cognitive impairment and physical functional impairment.

Due to the increased prevalence of diabetes and dementia as people age, the association between diabetes and cognitive impairment is increasingly being recognized. Previous studies suggested that diabetes patients had a higher risk of cognitive impairment. ${ }^{28-31}$ However, studies evaluating the associations of glycaemic control of diabetes with cognitive function in older adults are few. Similar to our results, the cross-sectional Mexican Health and Ageing Study of 216 older participants showed that poor glycaemic control was associated with worse global cognitive performance. ${ }^{11}$ Furthermore, a longitudinal study including 1289 American participants from the Alzheimer's Disease Neuroimaging Initiative suggested that untreated diabetes was associated with a higher risk for dementia, whereas treated diabetes was not, implying that the risk of diabetes-related dementia may be attenuated by glycaemic control. ${ }^{32}$ However, these studies had relatively smaller sample sizes, were of different ethnicities, did not make accurate dementia and dementia subtype diagnoses, or classified the participants with diabetes by self-reported antidiabetic agent status, without taking blood glucose levels into account. Inconsistent with our results, the Maine-Syracuse Longitudinal Study showed that glycaemic control was not associated with the cognitive function of diabetes patients. ${ }^{33}$ However, this study excluded participants with dementia, had a smaller sample size, and studied middle-aged to older adults, who were younger than our study, all of which may have led to the inconsistency.

Our study found no significant association of IFG with dementia, $\mathrm{AD}$, or global cognitive performance. A crosssectional population-based study of 2994 Swedish participants aged 61 years and older showed that prediabetes (defined as IFG or impaired glucose tolerance) was associated with worse global cognitive performance. ${ }^{34}$ However, it did not distinguish between IFG and impaired glucose tolerance, only analysed the associations between prediabetes and neuropsychological tests performance rather than dementia status, and it did not adjust for some potential confounders, such as APOE genotypes, which may have led to the inconsistency.

The mechanisms underlying the associations of diabetes with dementia and $\mathrm{AD}$ are likely to be multifactorial and bidirectional. On the one hand, diabetes induces neuronal death in different brain regions, 
especially the hippocampus, ${ }^{35}$ reduces the secretion of brain-derived neurotrophic factor, ${ }^{36}$ induces structural changes in cerebral microvessels, ${ }^{37}$ and leads to cerebrovascular disturbances. ${ }^{38}$ On the other hand, the accumulation of the amyloid-beta peptide, which is the main pathologic change in $\mathrm{AD}$, may induce neuronal insulin resistance, ${ }^{39}$ and insulin is significantly reduced in advanced $\mathrm{AD}$ brains compared with aged control brains. ${ }^{40}$ In addition, a person with dementia may be not able to properly participate in the process of diabetes therapy, which contributes to the poor diabetes control.

In addition to cognitive function, physical functioning is also a core element of health-related quality of life. The association between diabetes and physical functioning was studied in several population-based studies and the results are controversial. Consistent with our study, several studies have shown that diabetes is associated with worse physical functioning among older adults. ${ }^{14,41}$ However, unlike our study, the Health ABC Study included 2364 American older white and black adults and showed that although diabetes was associated with worse physical functioning, diabetes was not associated with the standing score, chair score, or walking score. ${ }^{42}$ The different ethnicities and different confounders for adjustment, such as peripheral nerve function, may have led to these different results. Despite the large number of studies on diabetes, only a limited number of studies have examined the association between diabetes and physical functioning in Chinese rural-dwelling older adults. Moreover, few studies have focused on the association between glycaemic control of diabetes and physical functioning.

Some mechanisms could explain the association between diabetes and physical functional impairment. Poor glycaemic control is associated with protein catabolism in skeletal muscle, which may lead to sarcopenia and thus result in a loss of physical function. ${ }^{41,43}$ Furthermore, insulin resistance impairs muscle glucose regulation, intracellular energy production and muscle contraction, which leads to a faster decline in muscle mass, strength and functional capacity. ${ }^{44}$

We did not find an association between IFG and physical functional impairment. Diabetes, especially diabetes with poor glycaemic control, was associated with a higher likelihood of cognitive impairment and physical functional impairment, but no association was detected between cognitive impairment or physical functional impairment and IFG. Therefore, early screening and effective treatment should be carried out at the IFG stage, and more attention should be paid to cognitive and physical function in older adults with diabetes.
Our study targeted rural-dwelling older Chinese adults, to whom inadequate attention has been given thus far. In addition, we used comprehensive and validated questionnaires to assess their dementia status and physical function. However, our study also has limitations. First, the cross-sectional nature of our study limited the possibility of making causal inferences. Second, we grouped the different glycaemic control statuses of diabetes by fasting blood glucose, since HbAlc was not available at baseline in the MIND-China Study. The standard for well-controlled diabetes may be too strict for for older adults, and the overtreatment of diabetes may also have a negative effect on cognitive functions and general fitness of elderly people with diabetes. ${ }^{45,46}$ Third, some detailed information on diabetes, such as diabetes type, duration, and complications, was not available. Fourth, the MMSE we used to evaluate the global cognitive function of older adults was largely influenced by the participants' educational level. For the illiterate persons, there was a significant increase in low scores of the MMSE, ${ }^{47}$ leading to a low level of MMSE score in our study. The "floor effect" may lead us to underestimate the associations between poor glycaemic control of diabetes and worse global cognitive function. Fifth, the study participants were recruited exclusively from a single rural area in China with limited education and low-to-medium socioeconomic status, which should be kept in mind when generalizing our findings to other populations.

\section{Conclusion}

In conclusion, this large population-based study adds to the body of evidence that poorly controlled diabetes is associated with all-cause dementia and AD. In addition, both well-controlled and poorly controlled diabetes were associated with physical functional impairment. IFG was not associated with dementia, global cognitive performance, or physical function. This also highlights that early intervention for IFG, which is at a high-risk state for developing diabetes, may be of great significance for the prevention of diabetes-related dementia, cognitive decline and physical functional impairment. Future longterm large-scale prospective studies and more comprehensive assessments of the glycaemic control status of diabetics are needed to clarify the causal relationships of IFG and the glycaemic control status of diabetes with cognitive and physical function. 


\section{Abbreviations}

$\mathrm{AD}$, Alzheimer's disease; ADLs, activities of daily living; APOE, Apolipoprotein E; ATC, Anatomical Therapeutic Chemical; BMI, body mass index; DSM-IV, Diagnostic and Statistical Manual of Mental Disorders, 4th Edition; HDL-C, high-density lipoprotein cholesterol; IFG, impaired fasting glucose; LDL-C, low-density lipoprotein cholesterol; MIND-China, Multimodal Intervention to Delay Dementia and Disability in Rural China; MMSE, Mini-Mental State Examination; NIA-AA, National Institute on Aging-Alzheimer's Association; NINDSAIREN, the National Institute of Neurological Disorders and Stroke and the Association Internationale pour la Recherche et l'Enseignement en Neurosciences; SPPB, Short Physical Performance Battery; TC, total cholesterol; TG, triglycerides; VaD, vascular dementia.

\section{Data Sharing Statement}

Data supporting the findings from this study will be available from the corresponding authors upon approval by the data management committee of MIND-China.

\section{Ethics Approval and Informed Consent}

MIND-China was approved by the Ethics Committee of Shandong Provincial Hospital in Jinan, Shandong. Written informed consent was obtained from all participants, or in the case of severely cognitively impaired participants, from informants. MIND-China was registered in the Chinese Clinical Trial Registry (registration no.: ChiCTR1800017758).

\section{Consent for Publication}

All authors confirm that the work described has not been published before; that it is not under consideration for publication elsewhere; and that its publication has been approved by all co-authors.

\section{Acknowledgments}

We would like to thank the study participants of MINDChina and all staff in the MIND-China research group for their invaluable contributions.

\section{Author Contributions}

All authors contributed to data analysis, drafting or revising the article, gave final approval of the version to be published, agreed to the submitted journal, and agreed to be accountable for all aspects of the work.

\section{Funding}

MIND-China was supported by grants from the National Key Research and Development Program of China (grant no.: 2017YFC1310100), the National Natural Science Foundation of China (NSFC, grants no.: 81861138008, and 82011530139), the Academic Promotion Program of Shandong First Medical University, and the Taishan Scholar Program of Shandong Province, China. Tang S received grants from the NSFC (grant no.: 82001397), and the Jinan Science and Technology Bureau (grant no.: 202019187).

\section{Disclosure}

The authors report no conflicts of interest in this work.

\section{References}

1. Cho NH, Shaw JE, Karuranga S, et al. IDF diabetes atlas: global estimates of diabetes prevalence for 2017 and projections for 2045 . Diabetes Res Clin Pract. 2018;138:271-281.

2. Wojszel ZB, Magnuszewski L. Type 2 diabetes correlates with comorbidity and nutritional status but not with functional health in geriatric ward patients: a cross-sectional study in Poland. Diabetes Metabol Syndr Obesity. 2020;13:4599-4607.

3. Wang L, Gao P, Zhang M, et al. Prevalence and ethnic pattern of diabetes and prediabetes in China in 2013. JAMA. 2017;317 (24):2515-2523.

4. Tabák AG, Herder C, Rathmann W, Brunner EJ, Kivimäki M. Prediabetes: a high-risk state for diabetes development. Lancet. 2012;379(9833):2279-2290.

5. Gerstein HC, Santaguida P, Raina P, et al. Annual incidence and relative risk of diabetes in people with various categories of dysglycemia: a systematic overview and meta-analysis of prospective studies. Diabetes Res Clin Pract. 2007;78(3):305-312.

6. Fei M, Yan Ping Z, Ru Juan M, Ning Ning L, Lin G. Risk factors for dementia with type 2 diabetes mellitus among elderly people in China. Age Ageing. 2013;42(3):398-400.

7. Thomassen JQ, Tolstrup JS, Benn M, Frikke-Schmidt R. Type-2 diabetes and risk of dementia: observational and Mendelian randomisation studies in 1 million individuals. Epidemiol Psychiatr Sci. 2020;29:e118.

8. Gudala K, Bansal D, Schifano F, Bhansali A. Diabetes mellitus and risk of dementia: a meta-analysis of prospective observational studies. J Diabetes Investig. 2013;4(6):640-650.

9. Cholerton B, Baker LD, Craft S. Insulin resistance and pathological brain ageing. Diabet Med. 2011;28(12):1463-1475.

10. Nguyen TT, Ta QTH, Nguyen TKO, Nguyen TTD, Giau VV. Type 3 diabetes and its role implications in Alzheimer's disease. Int $J \mathrm{Mol}$ Sci. 2020;21(9):3165.

11. Mimenza-Alvarado AJ, Jimenez-Castillo GA, Yeverino-Castro SG, et al. Effect of poor glycemic control in cognitive performance in the elderly with type 2 diabetes mellitus: the Mexican Health and Aging Study. BMC Geriatr. 2020;20(1):424.

12. Tolppanen AM, Lavikainen P, Solomon A, et al. History of medically treated diabetes and risk of Alzheimer disease in a nationwide case-control study. Diabetes Care. 2013;36(7):2015-2019.

13. Legrand D, Vaes B, Mathei C, Adriaensen W, Van Pottelbergh G, Degryse JM. Muscle strength and physical performance as predictors of mortality, hospitalization, and disability in the oldest old. $J \mathrm{Am}$ Geriatr Soc. 2014;62(6):1030-1038. 
14. Chiles NS, Phillips CL, Volpato S, et al. Diabetes, peripheral neuropathy, and lower-extremity function. J Diabetes Complications. 2014;28(1):91-95.

15. Ferrucci L, Penninx B, Leveille S, et al. Characteristics of nondisabled older persons who perform poorly in objective tests of lower extremity function. J Am Geriatr Soc. 2000;48(9):1102-1110.

16. Chau P, Woo J, Lee C, et al. Older people with diabetes have higher risk of depression, cognitive and functional impairments: implications for diabetes services. J Nutr Health Aging. 2011;15(9):751-755.

17. Cong L, Ren Y, Hou T, et al. Use of cardiovascular drugs for primary and secondary prevention of cardiovascular disease among rural-dwelling older Chinese adults. Front Pharmacol. 2020;11:608136.

18. Han X, Jiang Z, Li Y, et al. Sex disparities in cardiovascular health metrics among rural-dwelling older adults in China: a population-based study. BMC Geriatr. 2021;21(1):158.

19. Kivipelto M, Mangialasche F, Snyder HM, et al. World-wide FINGERS network: a global approach to risk reduction and prevention of dementia. Alzheimers Dement. 2020;16(7):1078-1094.

20. World Health Organization \& International Diabetes Federation. Definition and Diagnosis of Diabetes Mellitus and Intermediate Hyperglycaemia: Report of a WHO/IDF Consultation. Geneva, Switzerland: WHO; 2006.

21. Folstein MF, Folstein SE, McHugh PR. "Mini-mental state". A practical method for grading the cognitive state of patients for the clinician. J Psychiatr Res. 1975;12(3):189-198.

22. DAPA. Diagnostic and Statistical Manual of Mental Disorders. Washington, DC: American Psychiatric Association; 1994.

23. Sperling RA, Aisen PS, Beckett LA, et al. Toward defining the preclinical stages of Alzheimer's disease: recommendations from the National Institute on Aging-Alzheimer's Association workgroups on diagnostic guidelines for Alzheimer's disease. Alzheimers Dement. 2011;7(3):280-292.

24. Roman GC, Tatemichi TK, Erkinjuntti T, et al. Vascular dementia: diagnostic criteria for research studies. Report of the NINDS-AIREN International Workshop. Neurology. 1993;43(2):250-260.

25. Guralnik JM, Simonsick EM, Ferrucci L, et al. A short physical performance battery assessing lower extremity function: association with self-reported disability and prediction of mortality and nursing home admission. J Gerontol. 1994;49(2):M85-M94.

26. Mickute M, Henson J, Rowlands AV, et al. Device-measured physical activity and its association with physical function in adults with type 2 diabetes mellitus. Diabet Med. 2020;38:e14393.

27. Sun H, Ren X, Chen Z, et al. Association between body mass index and mortality in a prospective cohort of Chinese adults. Medicine (Baltimore). 2016;95(32):e4327.

28. Haroon NN, Austin PC, Shah BR, Wu J, Gill SS, Booth GL. Risk of dementia in seniors with newly diagnosed diabetes: a population-based study. Diabetes Care. 2015;38(10):1868-1875.

29. Ahtiluoto S, Polvikoski T, Peltonen M, et al. Diabetes, Alzheimer disease, and vascular dementia: a population-based neuropathologic study. Neurology. 2010;75(13):1195-1202.

30. Zhang J, Chen C, Hua S, et al. An updated meta-analysis of cohort studies: diabetes and risk of Alzheimer's disease. Diabetes Res Clin Pract. 2017;124:41-47.

31. Wang KC, Woung LC, Tsai MT, Liu CC, Su YH, Li CY. Risk of Alzheimer's disease in relation to diabetes: a population-based cohort study. Neuroepidemiology. 2012;38(4):237-244.
32. McIntosh EC, Nation DA. Importance of treatment status in links between type 2 diabetes and Alzheimer's disease. Diabetes Care. 2019;42(5):972-979.

33. Dore GA, Elias MF, Robbins MA, Elias PK, Nagy Z. Presence of the APOE epsilon4 allele modifies the relationship between type 2 diabetes and cognitive performance: the Maine-Syracuse Study. Diabetologia. 2009;52(12):2551-2560.

34. Dybjer E, Nilsson PM, Engstrom G, Helmer C, Nagga K. Prediabetes and diabetes are independently associated with adverse cognitive test results: a cross-sectional, population-based study. BMC Endocr Disord. 2018;18(1):91.

35. Machida M, Fujimaki S, Hidaka R, Asashima M, Kuwabara T. The insulin regulatory network in adult hippocampus and pancreatic endocrine system. Stem Cells Int. 2012;2012:959737.

36. Kim OY, Song J. The importance of BDNF and RAGE in diabetes-induced dementia. Pharmacol Res. 2020;160:105083.

37. Rom S, Zuluaga-Ramirez V, Gajghate S, et al. Hyperglycemia-driven neuroinflammation compromises BBB leading to memory loss in both diabetes mellitus (DM) type 1 and type 2 mouse models. Mol Neurobiol. 2019;56(3):1883-1896.

38. Riederer P, Korczyn AD, Ali SS, et al. The diabetic brain and cognition. J Neural Transm. 2017;124(11):1431-1454.

39. Sato N, Morishita R. Plasma abeta: a possible missing link between Alzheimer disease and diabetes. Diabetes. 2013;62(4):1005-1006.

40. Rivera EJ, Goldin A, Fulmer N, Tavares R, Wands JR, de la Monte SM. Insulin and insulin-like growth factor expression and function deteriorate with progression of Alzheimer's disease: link to brain reductions in acetylcholine. $J$ Alzheimers Dis. 2005;8 (3):247-268.

41. Volpato S, Blaum C, Resnick H, Ferrucci L, Fried L, Guralnik J. Comorbidities and impairments explaining the association between diabetes and lower extremity disability: the Women's Health and Aging Study. Diabetes Care. 2002;25(4):678-683.

42. Strotmeyer ES, de Rekeneire N, Schwartz AV, et al. The relationship of reduced peripheral nerve function and diabetes with physical performance in older white and black adults: the Health, Aging, and Body Composition (Health ABC) study. Diabetes Care. 2008;31(9):1767-1772.

43. Leenders M, Verdijk LB, van der Hoeven L, et al. Patients with type 2 diabetes show a greater decline in muscle mass, muscle strength, and functional capacity with aging. J Am Med Dir Assoc. 2013;14 (8):585-592.

44. Burton DGA, Faragher RGA. Obesity and type-2 diabetes as inducers of premature cellular senescence and ageing. Biogerontology. 2018;19(6):447-459.

45. Wojszel ZB, Kasiukiewicz A. A retrospective cross-sectional study of type 2 diabetes overtreatment in patients admitted to the geriatric ward. BMC Geriatr. 2019;19(1):242.

46. Wojszel ZB, Kasiukiewicz A. A retrospective time trend study of diabetes overtreatment in geriatric patients. Diabetes Metabol Syndr Obesity. 2019;12:2023-2032.

47. Katzman R, Zhang MY, Ouang YQ, et al. A Chinese version of the Mini-Mental State Examination; impact of illiteracy in a Shanghai dementia survey. J Clin Epidemiol. 1988;41(10):971-978. 


\section{Publish your work in this journal}

Clinical Interventions in Aging is an international, peer-reviewed journal focusing on evidence-based reports on the value or lack thereof of treatments intended to prevent or delay the onset of maladaptive correlates of aging in human beings. This journal is indexed on PubMed Central, MedLine, CAS, Scopus and the Elsevie
Bibliographic databases. The manuscript management system is completely online and includes a very quick and fair peer-review system, which is all easy to use. Visit http://www.dovepress.com/ testimonials.php to read real quotes from published authors. 